\title{
AKARI-SDSS-GALEX SURVEYS: SPECTRAL ENERGY DISTRIBUTIONS OF NEARBY GALAXIES
}

\author{
V. Buat ${ }^{1}$, F. -T. Yuan ${ }^{2}$, T. T. Takeuchi ${ }^{2}$, E. Giovannoli ${ }^{1}$, And S. Heinis ${ }^{1}$ \\ ${ }^{1}$ Laboratoire d'Astrophysique de Marseille - LAM, Université Aix-Marseille \& CNRS, UMR7326, 38 rue F. \\ Joliot-Curie, 13388 Marseille Cedex 13, France \\ ${ }^{2}$ Division of Particle and Astrophysical Sciences, Nagoya University, Furo-cho, Chikusa-ku, Nagoya 464-8602, \\ Japan \\ E-mail: veronique.buat@oamp.fr \\ (Received May 25, 2012; Accepted Jun 30, 2012)
}

\begin{abstract}
A sample of nearby galaxies was built from the AKARI/FIS all sky survey cross-correlated with the SDSS and GALEX surveys. The spectral energy distributions from 0.15 to 160 microns of these galaxies are analysed to study dust attenuation and star formation properties. The calibrations of the amount of dust attenuation as a function of the IR-to-UV flux ratio and the FUV-NUV colour are re-investigated: the former one is confirmed to be robust and accurate whereas the use of the FUV-NUV colour to measure dust attenuation is found highly uncertain. The current star formation rate given by the SED fitting process is compared to that directly obtained from the UV and total IR luminosities. It leads to an accurate estimate of dust heating by old stars. We emphasize the importance of such a sample as a reference for IR selected star forming galaxies in the nearby universe
\end{abstract}

Key words: infrared: galaxies; ultraviolet: galaxies; dust: extinction

\section{INTRODUCTION}

The nearby universe remains the best laboratory to understand the physical properties of galaxies and is a reference for any comparison with high redshift observations. The all sky (or very large) surveys that have been performed from the ultraviolet (UV) to the far-infrared (far-IR) provide us with large datasets and very large wavelength coverage to perform a reference study. The broad-band spectral energy distribution (SED) of a galaxy represents the combination of the emission from both stars of all ages and interstellar dust that interact in a complex way by means of the absorption and scattering of the stellar light by dust grains. The stars emit from the UV to the near-IR whereas the mid and far-IR emission comes from interstellar dust heated by the stellar emission. By comparing data with models, one can attempt to derive some physical parameters related to the star formation his- tory and dust attenuation in a homogeneous way and simultaneously for all galaxies of a given sample. With the availability of mid and far-IR data for large samples of galaxies, we can combine stellar and dust emission to analyse SEDs and deduce accurate star formation rates (SFRs).

We investigate the properties of nearby galaxies by selecting a sample of objects that is as large as possible, observed as part of the $A K A R I$, SDSS, and GALEX surveys. It corresponds to 13 photometric bands from 0.15 to $160 \mu \mathrm{m}$. Particular care is taken to measure total flux densities for the extended sources. Our aim is to perform SED fitting to deduce physical properties in a homogeneous and consistent way, focusing on dust attenuation and SFR measurements. The analysis and results presented here are published in Takeuchi et al. (2010), Buat et al. (2011) and Yuan et al. (2011). 


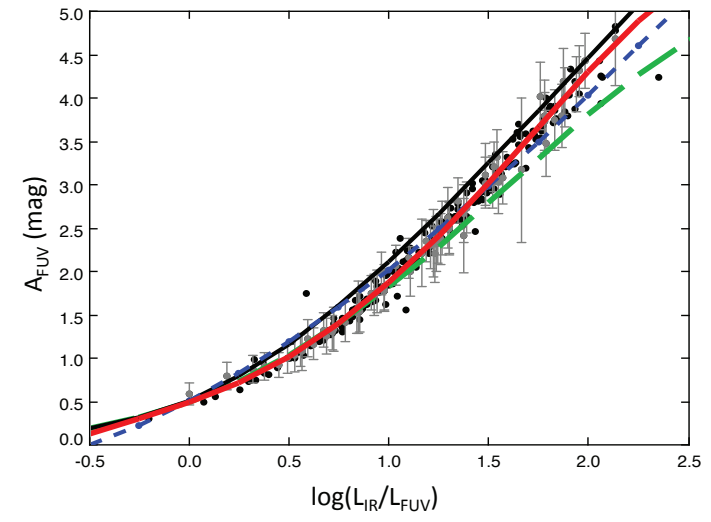

Fig. 1. $A_{\mathrm{FUV}}$ plotted as a function of $L_{\mathrm{IR}} / L_{\mathrm{FUV}}$, the result of the polynomial regression being plotted as a red thick solid line. Models from Meurer et al. (1999, black thin solid line), Buat et al. (2005, blue dotted line), and Cortese et al. (2008) with $\tau=6.2$ Gyr (green dashed line) are overplotted. Standard errors in $A_{\mathrm{FUV}}$ are plotted for $1 / 4$ of the objects.

\section{DATA}

Takeuchi et al. (2010) cross-matched the first primary catalogue of the $A K A R I /$ FIS All Sky Survey ( $\beta$-1 version) with the IRAS/PSCz to select 776 sources confirmed as galaxies, with a known redshift and observed with GALEX. For 607 of these 776 sources, SDSS/DR7 images are suitable for performing accurate photometry. Buat et al. (2011) restricted the sample to galaxies whose $140 \mu \mathrm{m}$ flux is of high quality, 363 sources fulfilling this condition. Most of selected sources (357 sources) also have high quality fluxes at $90 \mu \mathrm{m}$ but the fraction of these sources with reliable fluxes at 65 and $160 \mu \mathrm{m}$ is about $\sim 1 / 3$. Yuan et al. (2011) analysed the sub-sample of 153 galaxies matched with the AKARI/IRC PSC.

\subsection{Dust Attenuation}

An efficient way to derive physical parameters of star formation and dust attenuation homogeneously is to fit the observed SED with models from a stellar population synthesis code. We use the code CIGALE (Code Investigating GALaxy Emission) ${ }^{1}$, which derives physical information about galaxies by fitting their UV-tofar-IR SED with a full conservation of the energy budget (Noll et al., 2009; Giovannoli et al., 2011).

\footnotetext{
1 http://www.oamp.fr/cigale.
}

Dust attenuation in UV $\left(A_{\mathrm{FUV}}\right)$ is found to be tightly related to the ratio of IR to UV luminosity, $\log \left(L_{\mathrm{IR}} / L_{\mathrm{FUV}}\right)$. Applying a polynomial regression on our data yields (Fig. 1):

$A_{\mathrm{FUV}}=0.483+0.812 y+0.373 y^{2}+0.299 y^{3}-0.106 y^{4}$ where $y=\log \left(L_{\mathrm{IR}} / L_{\mathrm{FUV}}\right)$.

We also compare the values of $A_{\text {FUV }}$ obtained from our SED fitting method with the $F U V-N U V$ colour from GALEX.

The values are reported in Fig. 2 and exhibit a fairly weak correlation (correlation coefficient $\mathrm{R}=0.59$ ), a linear regression indicating that:

$A_{\mathrm{FUV}}=2.39( \pm 0.20) \times(F U V-N U V)+0.89( \pm 0.13)$

with $\sigma=0.83$. Several authors proposed a relation between $A_{\mathrm{FUV}}$ and $F U V-N U V$. Meurer et al. (1999) obtained a linear relation between the slope of the UV continuum $\beta$ and $A_{F U V}$ for starburst galaxies, Seibert et al. (2005) found that the starburst relation overestimates $A_{\mathrm{FUV}}$ by $\sim 0.5 \mathrm{mag}$ for a sample of galaxies not necessarily starbursting. These relations are reported in Fig. 2. We find that the relation proposed by Seibert et al. (2005) matches our results more closely than that of Meurer et al. (1999) but, in most cases, it overestimates the dust attenuation for a given $F U V-N U V$ colour. Salim et al. (2007) derived a shallower relation by fitting UV-optical data only (without IR data) for normal blue galaxies. Their relation is also reported in Fig. 2 and appears consistent with our own regression. In all cases the dispersion in the data leads to a standard deviation around the regression line of 0.8 mag. The large dispersion and the shallower relation than that found for starburst galaxies is explained by an attenuation curve only slightly steeper than that for starbursts (Calzetti et al., 2000) and variations of the star formation history. Note that the steepness of the relation between $F U V-N U V$ and $A_{\mathrm{FUV}}$ make the derivation of a dust attenuation from the $F U V-N U V$ color quite uncertain.

\subsection{Star Formation Calibrations}

Yuan et al. (2011) derived SFR calibrations using the S9W and L18W bands of the AKARI/ Infrared Camera (IRC). SFRs are obtained by fitting the whole SEDs with CIGALE and then are compared to the observed luminosities in the S9W and L18W bands. Tight relations are found as shown in Fig. 3 and 4 . Linear 


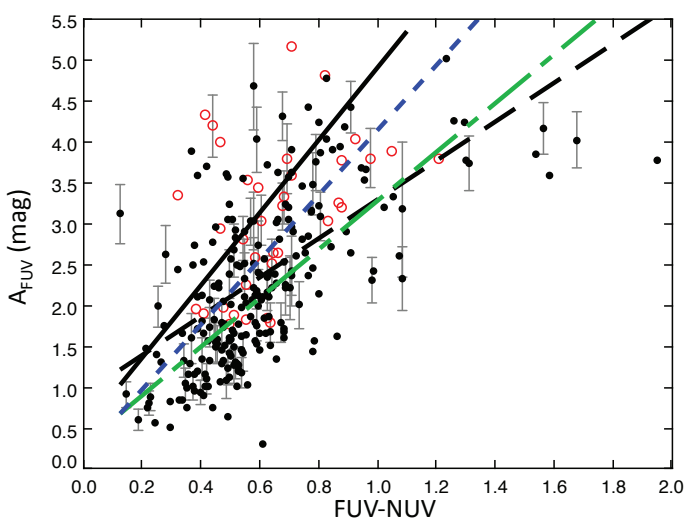

Fig. 2. $A_{\mathrm{FUV}}$ plotted against $F U V-N U V$. The linear regression is plotted as a dashed black line. Galaxies with $L_{\mathrm{IR}}>10^{11} L_{\odot}$ are plotted as empty red circles. Relations from previous studies are overplotted: Salim et al. (2007, green dot-dashed line), Seibert et al. (2005, blue dotted line), and Meurer et al. (1999, black solid line). Standard errors in $A_{\mathrm{FUV}}$ are plotted for $1 / 4$ of the objects.

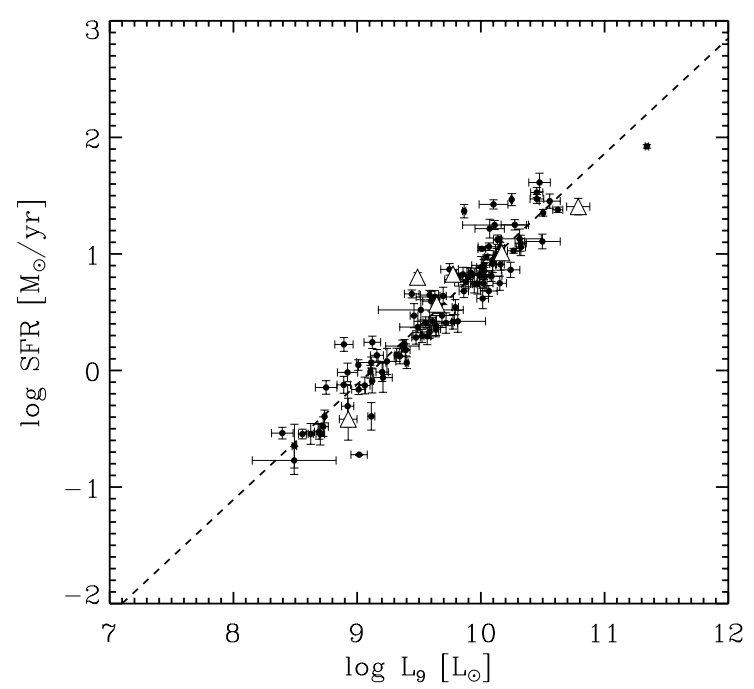

Fig. 3. Star formation rates from SED fitting plotted against the luminosity at $9 \mu \mathrm{m}$. The dashed line is the result of the regression. AGNs are plotted with triangles.

regressions give:

$$
\log \left(S F R_{M_{\odot} y r^{-1}}\right)=0.99 \log \left(L_{9, L_{\odot}}\right)-9.02
$$

and

$$
\log \left(S F R_{M_{\odot} y r^{-1}}\right)=0.90 \log \left(L_{18, L_{\odot}}\right)-8.03
$$

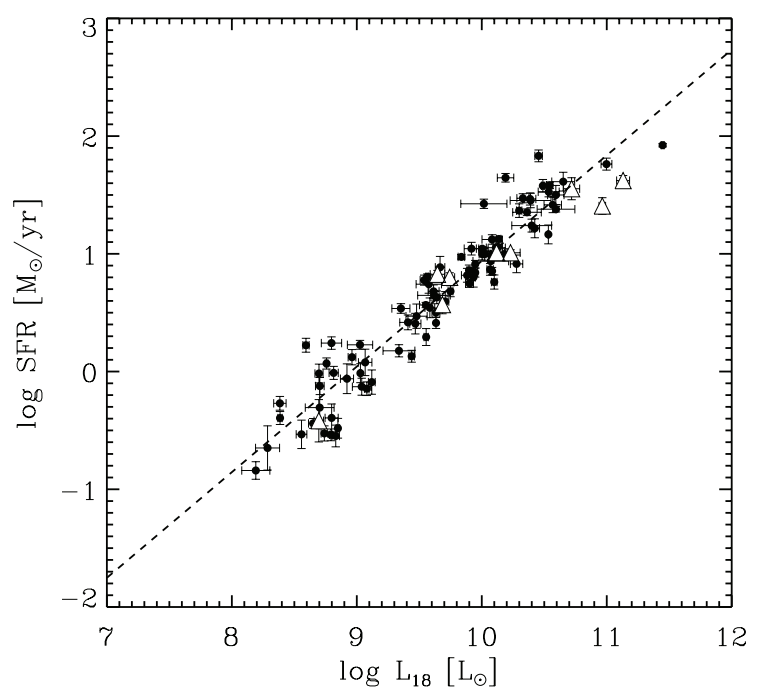

Fig. 4. Same as Fig. 6 with the luminosity at $18 \mu \mathrm{m}$.

The dispersion around the regression line is 0.2 dex. When both UV and IR fluxes are measured, a very efficient way to estimate SFR is to add the contributions of both types of emission to the total SFR. One must also account for dust heating by old stars, which is not directly related to the current star formation and given by

$$
\mathrm{SFR}_{\mathrm{tot}}=(1-\eta) \mathrm{SFR}_{\mathrm{IR}}+\mathrm{SFR}_{\mathrm{FUV}}
$$

where $\eta$ represents the fraction of IR emission due to dust heating by old stars, SFR $\mathrm{IR}$ being calculated by converting the total IR luminosity $L_{\mathrm{IR}}$ into SFR assuming that all the light from stars is absorbed by dust and $\mathrm{SFR}_{\mathrm{FUV}}$ is calculated from the observed UV luminosity $L_{\mathrm{FUV}}$. The relation between the IR and FUV luminosities and the corresponding SFRs are derived for a constant SFR over $10^{8}$ years (the typical duration of the UV emission to reach a steady state) and a Kroupa initial mass function (Buat et al., 2008). In Fig. 5, we report the variation in $\eta$ as a function of $L_{\mathrm{IR}}$ for our sample. $\langle\eta\rangle=0.17 \pm 0.10$ for the whole sample. A weak trend is found as a function of $L_{\mathrm{IR}}$ with $\langle\eta\rangle=0.14 \pm 0.08$ for $L_{\mathrm{IR}}>10^{11} L_{\odot}(26$ galaxies). Adopting $\eta=0.17$ leads to a very good agreement between $\mathrm{SFR}_{\text {tot }}$ and the SFR estimated by CIGALE. This agreement implies that the timescales over which both SFRs are calculated are similar.

The value of $\eta$ we find here is lower than that found by Hirashita et al. (2003) $(\eta=0.4)$. Bell (2003) estimated the mean contribution of old stellar populations to $L_{\mathrm{IR}}$ to be $32 \% \pm 16 \%$ for $L_{\mathrm{IR}}<10^{11} L_{\odot}$ and $9 \% \pm 5 \%$ for 


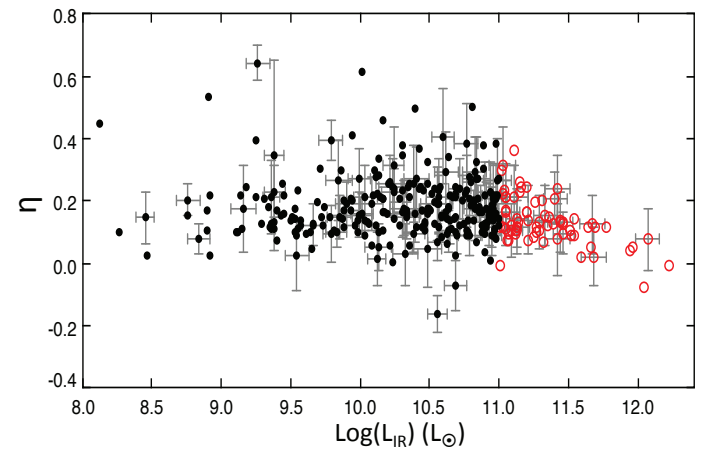

Fig. 5. Fraction of dust heating by old stars, $\eta$, plotted as a function of $L_{\mathrm{IR}}$, galaxies with $L_{\mathrm{IR}}>10^{11} L_{\odot}$ are plotted as empty red circles. Standard errors in both output parameters given by CIGALE are plotted as error bars for $1 / 4$ of the sample.

$L_{\mathrm{IR}}>10^{11} L_{\odot}$. These values and those of the present work are compatible within their uncertainties.

\section{ACKNOWLEDGEMENTS}

The Second AKARI Conference was supported by BK21 program to Seoul National University by the Ministry of Education, Science and Technology, Center for Evolution and Origin of Universe (CEOU) at Seoul National University, National Research Foundation Grant No. 2006-341-C00018 to HMLee, Astronomy Program, Seoul National University Nagoya University Global COE Program: Quest for Fundamental Principles in the Universe, Division of Particle and Astrophysical Science, Nagoya University, and Institute of Space and Astronautical Science, Japan Aerospace Exploration Agency. VB thanks the Centre National d'Etude Spatial for its financial support on the AKARIGALEX project

\section{REFERENCES}

Bell, E., 2003, Dust-induced Systematic Errors in Ultraviolet-Derived Star Formation Rates, ApJ 586,794

Buat, V., et al., 2005, Dust Attenuation in the Nearby Universe: A Comparison between Galaxies Selected in the Ultraviolet and in the Far-Infrared, ApJ, 619, L51

Buat, V., et al., 2008, Star Formation History of Galaxies from $\mathrm{z}=0$ to $\mathrm{z}=0.7$. A Backward Ap- proach to the Evolution of Star-Forming Galaxies, A\&A, 483, 107

Buat, V., et al., 2011, Spectral Energy Distributions of an AKARI-SDSS-GALEX Sample of Galaxies, A\&A, 529, 22

Calzetti, D., et al., 2000, The Dust Content and Opacity of Actively Star-forming Galaxies, ApJ, 533, 682

Cortese, C., et al., 2008, Ultraviolet Dust Attenuation in Star-Forming Galaxies - II. Calibrating the A(UV) Versus L(TIR)/L(UV) Relation, MNRAS, 386, 1157

Giovannoli, E., et al., 2011, Population Synthesis Modelling of Luminous Infrared Galaxies at Intermediate Redshift, A\&A, 525, 215

Hirashita, H., et al., 2003, Star Formation Rate in Galaxies from UV, IR, and $\mathrm{H} \alpha$ Estimators, A\&A, 410, 83

Meurer, G., et al., 1999, Dust Absorption and the Ultraviolet Luminosity Density at z 3 as Calibrated by Local Starburst Galaxies, ApJ, 521, 64

Noll, S., et al., 2009, Analysis of Galaxy Spectral Energy Distributions from Far-UV to Far-IR with CIGALE: Studying a SINGS Test Sample, A\&A, 507,1793

Salim, S., et al., 2007, UV Star Formation Rates in the Local Universe, ApJS, 173, 267

Seibert, M., et al., 2005, Testing the Empirical Relation between Ultraviolet Color and Attenuation of Galaxies, ApJ, 619, L55

Takeuchi, T. T., et al., 2010, Star Formation and Dust Extinction Properties of Local Galaxies from the AKARI-GALEX All-Sky Surveys . First Results from the Most Secure Multiband Sample from the Far-Ultraviolet to the Far-Infrared, A\&A, 514, A4

Yuan, F. T., et al., 2011, AKARI/IRC Broadband Mid-Infrared Data as an Indicator of the StarFormation Rate, PASJ, 63, 1207 